\title{
Les proverbes dans Kongo Proverbs and the Origins of Bantu Wisdom par Mukumbuta Lisimba
}

\author{
Ludwine Mabika Mbokou, Département d'afrikaans et de néerlandais, \\ Université de Stellenbosch, Stellenbosch, République d'Afrique du Sud \\ (13550721@humarga.sun.ac.za)
}

Résumé: On emploie tous les jours des proverbes, des maximes, des devinettes et des dictons. Malgré les progrès techniques des denières décénies en matière d'outils didactiques, le proverbe fait partie du quotidien des sociétés africaines en particulier et de celles du monde en général. Il reste un instrument d'éducation indispensable et vital pour les générations futures, et quiconque désire un conseil ou une orientation dans une certaine situation s'y réfère. Il fait partie intégrante de l'acte de communication.

À la lumière des analyses du Dr Lisimba de certains proverbes de la famille bantu, nous découvrons l'importance que revêt la dynamique du langage offert par les proverbes. Ils peuvent ainsi être employés comme données dans la compilation de dictionnaires. En effet, pour la discipline des sciences du langage qu'est la lexicographie, le traitement des proverbes et idiomes est d'une importance capitale parce que ces derniers sont le véhicule de la culture et des mœurs d'un peuple. Ainsi, pour mener à bien une telle entreprise, le lexicographe devra choisir le traitement à l'intérieur de la liste centrale, sinon à l'extérieur de la liste centrale, l'option la plus profitable pour lui restant une classification thématique qui tient compte non seulement de l'aspect sémantique du proverbe, mais aussi de son aspect formel.

Mots-clés: ANALYSE FORMELLE, ANALYSE SÉMANTIQUE, CLASSIFICATION THÉMATIQUE, DIDACTIQUE, DICTIONNAIRE, GENRE PROVERBIAL, IDIOMES, LEXICOGRAPHE, LEXICOGRAPHIE, LISTE CENTRALE, LITTÉRATURE ORALE, PRÉ-TEXTES, POST-TEXTES, PROVERBES

\begin{abstract}
Proverbs according to Kongo Proverbs and the Origins of Bantu Wisdom by Mukumbuta Lisimba. Proverbs, maxims, riddles and sayings are used every day. In spite of the technical progress during the last decades as far as didactic tools are concerned, the proverb forms part of the daily life of African societies in particular and those of the world in general. It remains an indispensable and essential educational instrument for future generations, and whenever someone wants advice or direction in a certain situation reference is made to it. It forms an integral part of the act of communication.

In the light of Dr Lisimba's analysis of certain proverbs of the Bantu family, the importance of the linguistic dynamics presented by proverbs will be discovered. They will therefore have to form part of the composition of dictionaries. In fact, for lexicography as discipline of linguistic science, the treatment of proverbs and idioms is of cardinal importance because they are the bearers of the culture and customs of a people. Therefore, to deal satisfactorily with proverbs, the lexicographer will have to choose a treatment either within or outside the main text, the most advantageous being
\end{abstract}


a thematic classification which allows not only for the semantic, but also for the formal aspects of the proverb.

Keywords: FORMAL ANALYSIS, SEMANTIC ANALYSIS, THEMATIC CLASSIFICATION, DIDACTICS, DICTIONARY, PROVERBIAL GENRE, IDIOMS, LEXICOGRAPHER, LEXICOGRAPHY, MAIN TEXT, ORAL LITERATURE, FRONT MATTER, BACK MATTER, PROVERBS

\section{Résumé de l'ouvrage}

Dans le domaine de la littérature orale, une attention particulière a souvent été portée aux proverbes. Il en résulte une abondante littérature dont la plupart des ouvrages sont des répertoires suivis et/ou précédés de brefs commentaires.

Tous les traveaux effectués sur les proverbes peuvent être répartis en deux groupes. D'une part il y a ceux dont le contenu se limite à une liste de proverbes suivis de l'explication et/ou d'une traduction de ce dernier. Ce sont pour la plupart des sortes de dictionnaires. Puis il y a les traveaux qui se focalisent sur la forme, le sens, l'origine et le contexte d'usage des proverbes. Les analyses effectuées dans ce genre de traveaux sont beaucoup plus linguistiques et sont davantage portées vers un public d'initiés. En effet, ces analyses ne se limitent pas seulement à dresser une liste de proverbes pour en démontrer la beauté stilistique et culturelle, mais elles constituent un développement pragmatique et fonctionnel du genre proverbial. C'est dans le même ordre de pensée que plusieurs ouvrages ont déjà été écrits sur la question des proverbes africains en général, et de manière plus particulière sur les proverbes appartenant à la famille de langue Bantu, avec une option spéciale pour la traduction et la classification thématique. La majorité de ces ouvrages ont eu pour support differents proverbes de tradition Bantu.

Dr Lisimba, chercheur et directeur du Centre International de Civilisation Bantu (CICIBA) s'inscrit dans cette lignée, avec une approche quelque peu différente de celle de ces contemporains. De par les analyses entreprises dans son ouvrage Kongo Proverbs and the Origin of Bantu Wisdom (Proverbes du Kongo et origine de la sagesse Bantu), le Dr Lisimba apporte certains changements en élargissant la description du proverbe Bantu par une étude comparative et contrastive. Une telle innovation repose sur le développement de la méthode ethnolinguistique, permettant ainsi une rigoureuse analyse formelle et sémantique.

Dans sa préface, Vatomene Kukanda pense que la méthode utilisée par le Dr Lisimba ne montre pas seulement comment se forme le proverbe, mais il retrace aussi les origines naturelles et psychologiques de la métaphore et du symbole dans ce dernier (Lisimba 1999).

\section{Approche proposée par l'auteur}

Comme l'indique le titre de l'ouvrage, le champ d'étude couvert par le Dr Li- 
simba est le domaine Bantu, plus précisement la zone Kongo. Son œuvre se compose de deux majeures parties. La première est théorique dans le sens où elle fait état de l'aspect fonctionnel et formel du proverbe. La seconde est un répertoire de proverbes de différents pays appartenant à cette zone. C'est dans la première partie de son ouvrage que sont examinées les caractéristiques fonctionnelles de ces derniers. Les deux sections de l'ouvrage constituent non seulement une approche nouvelle en ce qui concerne la description du proverbe dans le domaine Bantu, mais aussi une porte ouverte à toute autre discipline des sciences humaines et des sciences du langage. L'historien, le sociologue et l'anthropologue y trouveraient tout aussi bien leur compte que le sémanticien ou le syntacticien. En effet, chaque proverbe contient un thème dont il apporte une morale, un savoir.

Les analyses que fait le Dr Lisimba tiennent compte à la fois de la structure, de la fonction et du thème sous lequel le proverbe apparaît. Il choisit de faire une description des proverbes en basant son étude sur deux niveaux. Le niveau syntaxique où est compris l'aspect fonctionnel, et le niveau sémantique qui se concentre sur la structure fondamentale du sens du proverbe et sa fonction. La particularité qui en résulte se trouve dans le découpage en caractéristiques fonctionnelles et caractéristiques formelles. Ces dernières sont établies pour renforcer le choix de la classification thématique si souvent utilisée par les auteurs de traveaux ayant traits aux proverbes. Il rend cette classification plus explicite dans le sens où il ne répartit plus seulement les proverbes dans les différents thèmes, mais il inclut des critères de classification d'ordre sémantique. Dr Lisimba distingue par ailleurs deux genres de proverbes avec lesquels il faut compter pour une études explicite du sens des proverbes. La distinction qu'il pose a pour mérite d'amener à mieux cerner la signification des proverbes qui est souvent d'accès difficile.

En s'appuyant sur la définition donnée par l'Oxford Advanced Learner's Dictionary. Dr Lisimba présente le genre proverbial avec une identité double comprise entre sa forme et son sens. Il décrit ces deux critères comme étant cruciaux lorsqu'il s'agit de donner une définition générale du proverbe, et fondamentaux quant à l'aspect ethnolinguistique de l'approche.

C'est fort de cette ouverture que nous pensons que l'ouvrage du Dr Lisimba pourrait constituer une approche nouvelle et bénéfique dans le domaine lexicographique, en particulier en ce qui concerne le traitement des idiomes et proverbes dans un dictionnaire.

Notre attention pour cet article a été portée sur la première partie de l'ouvrage, dans laquelle nous avons trouvé des éléments essentiels permettant d'aboutir à des conclusions bénéfiques quant aux problèmes posés par les proverbes dans la compilation des dictionnaires. En effet, le lexicographe, face aux proverbes, se trouve confronté à plusieurs questions auxquelles il est parfois difficile de répondre. Questions qui sont étroitement liées à la définition du proverbe et à sa distinction avec les autres genres de la littérature orale tels que le mythe, la maxime, le conte ou la fable. Nous nous sommes donc intéressés 
aux quatre premiers chapitres de cette première partie qui présentent la description du proverbe dans les deux niveaux d'analyses mentionnés en sus. Pour le lexicographe qui a affaire aux proverbes lors de la compilation d'un dictionnaire, une telle approche est favorable et bénéfique. Nous le demontreront dans les lignes qui suivent en faisant une comparasion entre les principes préconisés par le Dr Lisimba et certaines démarches adoptées au cours de la compilation de dictionnaires.

\section{Presentation des chapitres}

L'auteur commence par une définition générale du genre proverbial. Cette définition implique la fonction et la forme du proverbe, ainsi que l'environnement dans lequel le proverbe est conçut, les destinataires et les utilisateurs de la communauté dont il est issu.

Le premier chapitre est un résumé du contenu de tous les chapitres qui suivront. Il sert d'introduction, et les différents points qui seront examinés tout au long des chapitres suivants y sont évoqués et clairement énoncés. Les chapitres deux et trois exposeront les analyses relatives à la classification thématique et la structure formelle du proverbe tandis que dans le chapitre quatre, l'aspect sémantique est développé.

\section{Le proverbe}

Dans les anciennes sociétés africaines à tradition orale, les proverbes constituaient un moyen d'instruction pour véhiculer toute la connaissance socioculturelle de la communauté. C'est de l'expérience de la vie humaine que les proverbes tirent leur source. Aujourd'hui encore, ils sont façonnés et modelés par la communauté linguistique qui les produit. Ils naissent de la société, en sont le produit, et sont par la même occasion utilisés par cette dernière de sorte qu'au fur et à mesure que les époques changent, les proverbes restent les mêmes (tout au moins dans leur fond).

Dans ces sociétés, "savoir parler" se rapportait à faire un usage habile des proverbes, et ce dans quelques circonstances que ce soit. Aujourd'hui encore, ils s'utilisent comme moyen d'instruction dans le sens où ils s'emploient lorsqu'il s'agit de véhiculer un savoir, communiquer une morale, ou prodiguer un conseil. Les proverbes traduisent la culture d'un peuple, d'une communauté linguistique quelle qu'elle soit. Ils représentent un moyen d'instruction et véhicule un savoir, particulièrement pour les communautés d'Afrique. Ils renferment les coutumes et mœurs d'un pays aussi bien qu'ils constituent un héritage pour les génération présentes et futures.

De plus, lorsqu'il s'agit de réflexion sur les principes de vie, sur des situations très difficiles à appréhender, ou encore lorsqu'il s'agit de choix à faire, on est souvent à la recherche de sages paroles qui mettraient de la lumière dans 
nos réflexions. Les proverbes par le savoir qu'ils véhiculent font jaillir la lumière quant à de telles situations. C'est dans ce sens que Akporobaro et Amovon (1994: 1) disent:

Of the varied literary forms, the proverb is the form which has proved itself to be of great continuing relevance to modern man. It has been, and remain a most powerful and effective instrument for the transmission of culture, social morality manners and ideas of a people from one generation to another. ${ }^{1}$

Exemple 1:

- Jette ton pain à la surface des eaux, avec le temps tu le retrouveras; se dit pour signifier à une personne qu'il est toujours utile de faire du bien autour de soi (proverbe de Salomon);

- Quand les singes et les écureuils s'amusent sur les branches, les brindilles tombent sur le dos des éléphants; se dit pour signifier que ce sont toujours les plus grands qui recçoivent les coups lorsqu'il y a des bévues (proverbe gabonais);

- Le léopard inspire toujours la crainte dans la forêt même lorsqu'il a perdu ses dents; se dit pour signifier qu'un chef reste un chef quelles que soient les circonstances (proverbe bakongo);

- Celui qui monte sur un arbre doit savoir distinguer une branche sèche d'une branche verte; se dit pour avertir à la prudence, ou pour inciter à la réflexion avant un projet (proverbe yipunu);

- L'abeille est honorée parce qu'elle travaille non pour elle seule, mais pour tous; se dit pour marquer les bénéfices de la générosité et du partage (proverbe chinois).

Dr Lisimba, dans son ouvrage, définit le proverbes comme étant essentiellement un état destiné à être appliqué à une situation sociale, dans un contexte présent. Maputa Mboukou, pour sa part, le définit par rapport à sa fonction dans la littérature orale: Parce que les proverbes traduisent les réalités de l'activité humaine par rapport à des situations actuelles et ponctuelles, il les décrit comme une situation qui véhicule une morale, mais à cette différence que cette situation n'est ni épique, ni une légende, mais un vécu.

Un proverbe ne peut donc être compris et/ou utilisé que dans un contexte bien précis. Les utilisateurs tout comme les destinataires de ce dernier doivent en connaitre le code. Les auteurs qui travaillent sur ce type de littérature doivent savoir comment en présenter le code afin de permettre aux lecteurs de le déchiffrer. À cette fin, ils doivent tenir compte du contexte d'utilisation dans lequel le proverbe est utilisé, de sa forme, et du genre auquel il appartient. En effet, on distingue plusieurs forme de proverbes qui vont des plus complexes aux simples proverbes phrases. Les proverbes phrases sont les plus facile à appréhender. Ils se retrouvent le plus souvent sous forme de phrases éliptiques et leur structure profonde diffère très peu de leur structure superficielle (Lisimba 1999: 77-88). Certains de ces proverbes sont utilisés pour exprimer des états de faits et sont des assertions soient positives, soient négatives. 
Exemple 2:

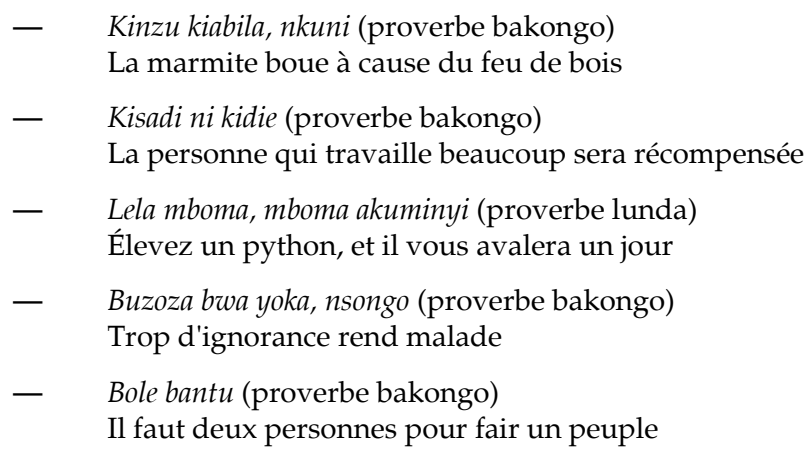

D'autres sont utilisés pour exprimer des cautions et des réprimandes. Dans ces derniers, la forme éliptique tend à disparaître. Les sociétés africaines utilisent ce type de proverbes pour parler des thèmes liés à l'éducation de l'enfant et la conduite d'une femme ou d'une jeune fille.

Exemple 3:

- $\quad$ A crab does not beget a bird (proverbe nigérian)

Une crabe ne donne pas naissance à un oiseau

- Kgomo go gatana tsa saka le le lengue (proverbe tswana)

Seules les vaches d'une même ferme se marchent dessus

- Musiru u wara na miri (proverbe yipunu)

La forêt se perpétue par ses arbres

- Mwana gè dji, mu bè odjougala (proverbe yipunu)

L'enfant qui refuse de manger doit être conduit dans un endroit désert

- Mwana wa munyako ngwako (proverbe tumbuka)

L'enfant de ton voisin est aussi le tien

Ces deux types de proverbes appartiennent à la première distinction faite par le Dr Lisimba, à savoir, les proverbes dont le thème est explicite à l'intérieur de la structure de surface. Ces derniers sont le plus souvent utilisés dans les dictionnaires comme exemples et illustrations. Ils ne nécessitent pas un traitement trop particulier car leur portée sémantique sert à renforcer le sens de notion abstraite comme la patience, la joie, l'amour.

Quant aux proverbes les plus complexes, ils sont ceux qui expriment des conseils et des directives. Leur structure superficielle est très différente de la structure profonde. Ils peuvent être constitués d'une ou de plusieurs phrases.

\section{Exemple 4:}

Ntsila gu saka dji gwè gnangu, go didjulu mabaga (proverbe yipunu)

En suivant un sentier en plein jour, on en reconnaît pas les bifurcations 


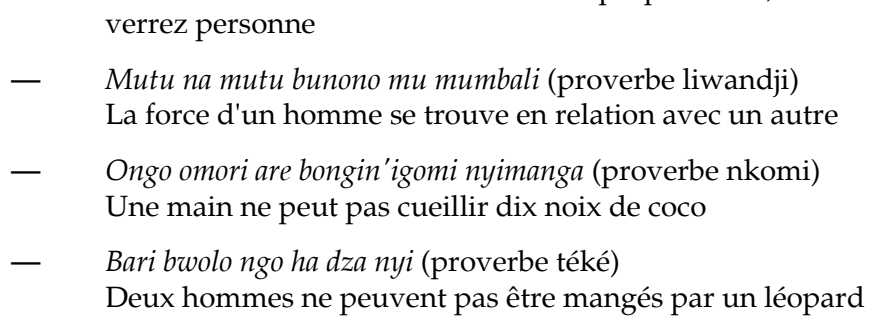

Dans les pages introductives d'un ouvrage sur les proverbes nigérians intitulé Nigerian Proverbs: Meaning and Relevance Today, les auteurs posent comme objectif principal la présentation du proverbe comme instrument actif de la société nigériane. Le soucis de ces derniers s'explique par la pensée qui fait du genre proverbial un fait démodé, dépassé. Pourtant, tous les jours, il se dit des proverbes, des dictons, des devinettes, des maximes (Maloux 1980: I). Malgré le développement des temps modernes, les proverbes ont traversés les âges et les époques. Et même la dépravation que subissent les mœurs dans ces dernières décénies apparaît comme un tremplin pour la promotion et l'utilisation des proverbes dans la vie communautaire, en général, et chez les jeunes en particulier. Les proverbes dans ce sens sont comme des gardes-fous.

Dr Kuzwayo, professeur de littérature orale sud-africaine, parle des proverbes comme une solution à la montée de violence et de délinquence juvénile à laquelle fait face son pays. Pour elle, ils sont les garants de la culture et de l'héritage des peuples. En tant que tel, ils protègent les valeurs morales et se chargent de les retransmettre aux générations futures.

As I grappled with the frightening violence and I racked my mind for remedies, I had to conclude that any effective remedy would have to combine a variety of solutions. And the language of proverbs struck me as one of the instruments, which could help. ${ }^{2}$

Dans la société africaine comme dans toutes autres sociétés, l'aspect géographique et environnemental influence les caractères utilisés dans les proverbes. Les personnifications d'êtres appartenant soit au règne animal, soit au règne végétal sont très prisées. Elles traduisent l'activité humaine de la communauté linguistique dans et pour laquelle les provebes sont conçus tout aussi bien que l'environnement, le climat, le relief, etc. On retrouvera ainsi des personnifications d'animaux de la savane, de la forêt, du désert, etc. Même l'aspect religieux sera pris en compte. Par exemple au Nigéria et au Cameroun, les proverbes utilisés par les populations du nord auront une influence provenant de l'islam parce qu'elle domine sur le plan culturel dans ces régions. Ainsi, le contenu d'un proverbe dépendra non seulement de la communauté linguistique qui l'utilise, mais aussi des mythes, des croyances et coutumes, et de l'environne- 
ment géographique de cette dernière. Pour bien comprendre et/ou expliquer un proverbe, il faut donc tenir compte de tous les aspects sus-mentionnés. En effet, la portée sémantique contenue à l'intérieur du proverbe ne saurait être appréciée dans sa totalité. Car n'oublions pas que c'est la vie de toute la société et les comportements humains qui sont représentés dans les proverbes. ${ }^{3}$

Un autre point à ajouter est que tout proverbe, ou du moins certains d'entre eux, porte un double manteau. Il peut véhiculer une assertion à la fois positive et négative par rapport au fait que les peuples diffèrent dans leur coutumes les uns des autres. Par conséquent, la compréhension des différents concepts traités dans les proverbes peut être différente d'une communauté à une autre, mais la fonction reste la même. Ils véhiculent tous une morale, un conseil, une sagesse, un savoir. C'est dans ce sens que Akporobaro et Emovon (1994: 10) disent:

The proverbs are crystallized forms of human experiences which are extremely diversified. Since there is no single solution to any one humain problem, proverbs are not offered as unique inviolables solution. (...) they present very clearly possible ready-made answers and advices to the multiplicity of human situations. ${ }^{4}$

\section{Exemple 5}

- Dress up the little piece of wood, it will become pretty (proverbe nigérian) Habillé, un vulgaire morceau de bois paraît beau.

Pour les nigérians, ce proverbe est employé pour montrer le ridicule, ou pour tourner en dérision. Dans la culture européenne par contre, il invite au courage, à l'optimisme. Tout peut être beau, il suffit de le vouloir. Ce proverbe se lira donc selon deux points de vue. D'une part, le point de vue négatif du nigérian qui l'utilisera pour montrer le grotesque d'une jeune fille laide qui prétend à la coquêterie, d'autre part, celui d'un européen pour qui ce sera le moyen de démontrer un tout autre point de vue: Un clochard peut paraître repoussant dans ses loques, mais vêtu de manière décente, il devient un prince charmant.

La double charge que peut porter le proverbe selon les cultures se retrouve aussi dans la symbolique de l'imagerie. Un tel animal peut représenter la force dans une société alors qu'il représente tout le contraire dans une autre.

\section{Les proverbes dans les dictionnaires}

Dans le domaine de la lexicographie pratique, le traitement des proverbes, s'il n'est pas bien organisé, entraîne une confusion chez le lecteur, beaucoup plus qu'il ne contribue à l'aider. Certains lexicographes, pour éviter ce genre de problèmes, préfèrent ne pas du tout les placer comme données (les proverbes sont des données de nature complexe). D'autres par contre les utilisent soit comme exemple, soit comme sens polysémique d'un lemme, voir même comme syno- 
nyme. La confusion qui peut se créer dans l'esprit du lecteur vient du fait que les proverbes sont traités dans les dictionnaires sans indications aucunes.

Tout proverbe se rapporte à un thème, un sens bien donné. Le recours à une classification thématique paraît alors plus judicieux pour le traitement des proverbes. Cette classification se fait au préable lors du décorticage des informations par le lexicographe. Elle constitue ainsi un début de réponse à la question de savoir quelle place donner aux proverbes et idiomes dans un dictionnaire. Partant du genre de classification proposée par le Dr Lisimba, nous avons essayé de proposer une adaptation qui permettrait d'améliorer les deux approches couramment utilisées en lexicographie pratique. Le lexicographe a le choix entre:

- traiter les proverbes à l'intérieur de la liste centrale, ou

- traiter les proverbes dans la structure textuelle de l'ouvrage.

Les dictionnaires ont longtemps été utilisés comme instruments dans l'acquisition et l'usage correct du language. L'un de leurs attributs est de donner une somme d'informations aux usagers pour leur permettre d'approfondir leurs connaissances. Ce sont des ouvrages de référence. Ils ont toujours joui d'une certaine autorité car ils permettent l'usage des mots, des constructions et des sens d'une langue en les intégrant dans la communauté. Ils leurs donnent ainsi le droit d'exister ou les condamnent en rejetant leur usage (Dubois et Dubois 1971: 51). Ils représentent par là même, la norme d'une langue. Cependant, ils demeurent moins rigides que les grammaires traditionnelles. Cette réalité du dictionnaire dans une communauté linguistique fait ressortir son caractère pédagogique. En effet, le système éducatif d'une société a pour objectif d'amener tout individu à se fondre dans une certaine culture, garant de la société dans laquelle il est appelé à vivre. Le dictionnaire transmet alors une série de règles avec lesquelles il doit compter pour ête reconnu comme membre de la communauté linguistique à laquelle il appartient.

De plus, le langage que véhicule le dictionnaire est par essence didactique. Il contribue donc à l'instruction de ceux qui l'utilisent.

Le dictionnaire est un outils fiable quant à la standardisation des langues. Par son aspect normatif, il en impose aux usagers parce qu'il offre, ou encore propose les règles d'usage des différents items lexicaux par leur traitement macro- et microstructurel. Conséquemment, le traitement et l'utilisation des proverbes dans les dictionnaires ne servent pas uniquement à préserver les proverbes, ou à les utiliser comme exemples, mais ils contribuent aussi à l'aspect pédagogique inhérent au fait de dictionnaire. Le lexicographe prévoit ainsi pour les générations présentes et futures un moyen d'instruction qui, dans l'Afrique moderne, tend à disparaître. Le dictionnaire permet alors de mettre à la disposition de tout locuteur, natif ou non-natif, un savoir universel. Car, bon nombres de proverbes partagent les mêmes thèmes, l'activité humaine étant la même de part et d'autre du globe terrestre. La portée sémantique et fonctionnelle du proverbe reste donc inchangée d'un continent à un autre, tandis que l'aspect formel varie. Par ailleurs, dans le dictionnaire, les informations qui sont 
traitées peuvent être d'ordre géographiques, historiques, voire même dialectal, la langue étant considérée comme la vision de la communauté linguistique qui la parle.

\section{Les proverbes à l'intérieur de la liste centrale}

Le traitement des proverbes comme exemples n'est pas une approche nouvelle, bien au contraire, c'est l'une des plus utilisées par les lexicographes. Elle revêt un caractère pratique en ce qu'elle satisfait au souci d'économie du lexicographe, c'est-à-dire, mettre le maximum d'informations à la disposition de l'usager, sans pour autant "gonfler" le contenu du dictionnaire. Elle constitue aussi un moyen de garage en ce sens qu'elle peut éviter au lexicographe de fournir trop d'explications quant à la portée sémantique du proverbe traité. Ce qui, dans certains cas, peut porter à confusion. Par exemple, dans les prétextes du Micro Robert (dictionnaire d'apprentissage du français), il est spécifié que les proverbes sont donnés en exemple et traités avec des étiquettes parce qu'il est nécessaire de connaître certaines tournures. Ils sont ainsi différenciés des exemples usuels que les auteurs de ce dictionnaire nomment exemples libres.

Il est aisé pour certains proverbes d'en retrouver le sens. Pour d'autres par contre, il faut toute une somme d'informations pour arriver à en déchiffer le code. Dans le traitement de ce genre de proverbe, le sens se trouve souvent confondu avec le sens premier du mot clé, et semble parfois sortir de nul part. Le locuteur ne sait pas si il a affaire à un sens polysémique, à un synonyme, à une locution, etc.

Maloux (1980: X) écrit que "tous les jours il se dit des proverbes". Pour lui, "ils jaillisent de la verve populaire". C'est cette même "verve" qui est le dénominateur commun entre les usagers et le dictionnaire. Ces derniers entendent et parlent le langage que le lexicographe est chargé de rendre dans le dictionnaire, parfois en rejetant, ou en approuvant l'usage. Et ce, de la manière la plus accessible qu'il soit. Le dictionnaire a aussi pour but de développer le langage de ceux qui le consultent. En utilisant la distinction proposée dans l'ouvrage du Dr Lisimba, le lexicographe peut ainsi sélectionner les proverbes les plus faciles d'accès, et souvent les plus fréquents, et les utiliser pour illustrer les définitions des notions abstraites telles l'amour, la patience, la joie, etc. Ce procédé est souvent utilisé dans les dictionnaires scolaires.

Les proverbes, comme mentionné en sus, prennent leur source dans l'observation du règne animal et végétal, et dans l'environnement immédiat de la communauté auxquels ils appartiennent. Dans le proverbe "petit à petit l'oiseau fait son nid", comme illustration de la définition du lemme "nid", il est à noter que le sens du proverbe n'a rien à voir avec la définition même du mot, mais plutôt avec le comportement de l'animal face à la confection de son habitat (ce dernier use de beaucoup de patience et prend le temps de chercher les brindilles qui conviennent à un tel ouvrage). Par contre, comme illustration ou exemple du lemme "patience", ou celui de "persévérence", il serait plus facile à l'usager non seulement de comprendre son sens, mais aussi de savoir l'utiliser dans 
un contexte approprié. D'où l'intérêt d'un traitement thématique tel que celui proposé par le Dr Lisimba.

Nous tenons à signaler qu'il est aussi possible d'avoir recours à une procédé autre que les exemples pour le traitement des proverbes à l'intérieur de la liste centrale. Il consiste à ouvrir une zone dans l'article de façon à traiter tous les proverbes découlant de l'item lexical retenu comme lemme dans la structure du dictionnaire. Cette approche est très prisée dans la compilation de dictionnaires élargis parce qu'elle est beaucoup plus appropriée. En effet elle permet de donner un nombre considérable d'informations sur un traitement "complet" de la langue étudiée. Ces informations qui doivent nécessairement être fournies par ce type de dictionnaire parce qu'il a pour but de traiter le vocabulaire de la langue étudiée dans sa quasi-totalité, tout en permettant d'en comprendre le fonctionnement. Ils sont plus descriptifs que la plupart des autres types de dictionnaires.

Bien que traités sous la bannière d'un lemme, les proverbes dans cette procédure le sont par rapport à leur sens. Nous n'avons donc pas choisi de présenter un examen complet de cette approche parce qu'elle ne saurait être adaptée aux analyses qui ont retenu notre attention. En outre présentent-elles très souvent un examen complet des proverbes dans les dictionnaires élargis.

Les proverbes dans la liste centrale peuvent encore être utilisés comme substitut dans la définition d'un lemme. Surtout lorsqu'il s'agit d'un terme abstrait. Mais dans ce cas comme dans celui du traitement séparé, il convient à un type bien défini de dictionnaire. Il est préférable de l'utiliser dans le cadre des dictionnaires bilingues et des dictionnaires de débutants.

\section{Les proverbes à l'extérieur de la liste centrale}

Pour cette approche, le procédé est le même que pour le traitement séparé à l'intérieur la liste centrale. La différence se trouve au niveau de la zone. Cette dernière se trouve généralement en fin de dictionnaire dans les post-textes, et non plus à l'intérieur de la liste centrale. Le plus souvent, cette zone est le lieu où le lexicographe choisit de placer les éléments de culture générale (cartes, noms de villes/pays, noms propres, citations, etc.) qu'il juge nécessaire à la connaissance du lecteur. Il les traite de manière séparée pour ne pas surcharger le contenu de la liste centrale et/ou en souligner l'importance. Sur cette lancée, les proverbes se trouvent dressés en répertoire et toujours par ordre alphabétique.

\section{Exemple 6}

À cour vaillant rien d'impossible, avec du courage, on vient à bout de tout.

$\grave{A}$ la Sainte-Luce, les jours croissent $d u$ saut d'une puce, les jours commencent à croître un peu à la Sainte-Luce (autref. 13 décembre, auj. 23 décembre).
À l'impossible nul n'est tenu, on ne peut exiger de quelqu'un ce qu'il lui est impossible de faire.

Bien mal acquis ne profite jamais, on ne peut jouir en paix du bien obtenu par des voies illégitimes. 
Bon chien chasse de race, on hérite généralement les qualités de sa famille.

Bonne renommée vaut mieux que ceinture dorée, mieux vaut jouir de l'estime publique que d'être riche.

Il n'y a que la vérité qui blesse, les reproches vraiment pénibles sont ceux que l'on a mérités.

Il n'y a que le premier pas qui coûte, le plus difficile en toute chose est de commencer.

Loin des yeux, loin du cour, l'absence détruit ou affaiblit les affections.

Les loups ne se mangent pas entre eux, les méchants ne cherchent pas à se nuire.
Mauvaise herbe pousse toujours, se dit pour expliquer la croissance rapide d'un enfant de mauvais caractère.

Qui aime bien châtie bien, un amour véritable est celui qui ne craint pas d'user d'une sage sévérité.

Rira bien qui rira le dernier, qui se moque d'autrui risque d'être raillé à son tour si les circonstances changent.

Si jeunesse savait, si vieillesse pouvait, les jeunes manquent d'expérience, les vieillards de force.

Tel père, tel fils, le plus souvent, le fils tient de son père.

Le temps, c'est de l'argent, traduction de l'adage anglais Time is money, le temps bien employé est un profit.

(extrait tiré du Dictionnaire de Français Contemporain)

Ce genre de procédure est beaucoup plus astucieux que de traiter les proverbes comme exemples, surtout lorsqu'il s'agit de dictionnaires pédagogiques. Elle permet en effet un traitement complet des proverbes, en donnant le maximum d'informations à tous les niveaux (informations d'ordre sémantiques, culturels, formels, etc.). Elle joue dans un certain sens le rôle de la mini-grammaire dans un dictionnaire. Cependant, dans un soucis d'économie et pour un rendement meilleur de la production, il serait préférable pour le lexicographe, de laisser de côté l'ordre traditionnel alphabétique, et de procéder par classification thématique. Car, plusieurs proverbes peuvent partager le même thème; dans ce cas le contexte d'usage est l'un des facteurs qui permet de saisir la distinction sémantique de chacun. Puis, il y a des proverbes qui se retrouvent sous la banière de plusieurs thèmes à la fois. Traiter les proverbes dans les post-textes d'un dictionnaire offre donc un plus large champ d'action. La présentation qui y est faite se révèle plus explicite, et s'avère plus claire et plus utile pour l'usager.

De plus, cette façon de traiter les proverbes nécessite plus d'effort de la part du lecteur, et parfois même du lexicographe parce que le fait de partager le même "mot-clé" n'a généralement rien à voir avec la portée sémantique du proverbe (comme avec l'exemple de "nid" dans "petit à petit l'oiseau fait son nid"). Le lecteur doit alors s'engager dans un tri pénible pour retrouver l'information dont il a besoin (rappelons au passage que les usagers des dictionnaires ne lisent pas les directions d'usage proposées par les auteurs dans les pré-textes). L'exemple 7 qui suit en est une illustration. Les auteurs de ce dictionnaire bilingue de proverbes ne présentent aux usagers qu'une somme de proverbes liés au lemme principal. Par exemple, aucun contexte d'usage n'est proposé, et 
la distinction entre le sens du proverbe et la définition du lemme, s'il y en a une, est inexistante. Les auteurs comptent avec l'intuition des usagers, ce qui est parfois trompeur dans le domaine de la lexicographie pratique.

\section{Exemple 7}

\section{FATHER:}

To be gathered to one's fathers: To die: Tot jou vaders vergader wees.

LIKE father, like SON; like mother, like daughter: Resembling the parents: So vaartjie, so moertjie.

\section{FAVOUR:}

To COUNT in one's favour: To be regarded as a merit: Ten goede kom; tot voordeel strek; as verdienste toereken.

To FIND favour with: To meet with approval: Genade vind; instemming vind.

IN favour of: On behalf of; in support of; to the advantage of: Ten gunste van.

To be OUT of favour: To have lost the goodwill of the others: Uitgebak wees; in onguns wees.

To WIN (general) favour: To become popular: Gewild word; die goedkeuring wegdra; opgang maak.

To favour SOMEONE: To treat someone with partiality: Iemand begunstig; vir iemand party trek.

\section{FEATHER:}

A feather in one's CAP: Something one may be proud of: ' $n$ Veer in jou hoed.

That is a feather in his CAP: That is something he can be proud of: Dis ' $n$ pluimpie vir hom.

To have one's feathers CROPPED: To be humiliated: Verneder word; sy stertvere kwyt wees.

FINE feathers make fine birds: People are judged by their outward appearance: Die klere maak die man; die vere maak die voël.

To be in HIGH feather: To be in good spirits: In 'n goeie bui wees; in jou noppies voel (wees).

You could have KNOCKED me down with a feather: I was most surprised: Jy kon my omgeblaas het; ek was skoon verbaas.

As LIGHT as a feather: Having little weight: So lig soos ' $n$ veer.

To show the WHITE feather: To be a coward: Lafhartig wees; papbroekig wees.

As you feather your NEST, so you must lie on it: You must accept what you have been responsible for: Wat jy saai, sal jy maai.

(extrait tiré d'English Proverbs and Expressions with Afrikaans equivalents)

\section{Perspectives et conclusion}

Un bon dictionnaire est un dictionnaire qui présente ses données de manière à ce que le l'usager en comprenne le sens et l'utilisation. L'idéal serait pour le lexicographe que les usagers se réfèrent aux pré-textes pour savoir comment se 
diriger dans la voie qui les conduiraient aux informations dont ils ont besoin. Bien de problèmes seraient alors vite résolus. Malheureusement, ce n'est pas toujours le cas. Il arrive même très rarement que les usagers passent par les pré-textes avant de consulter la liste centrale. Ils ne se préoccupent même pas de leur utilité. Cette situation est encore plus dramatique lorsqu'il s'agit de lecteur tels que les élèves, les étudiants. La présentations des informations à l'intérieur du dictionnaire revêt alors un caractère capital dans le travail du lexicographe.

Nous avons exposé dans nos propos l'esquisse d'une approche profitable des proverbes quant à leur traitement dans la lexicographie pratique. Les différents procédés examinés nous ont amené à proposer la classification thématique proposée par le Dr Lisimba comme un moyen plus tenable. Voici quelques avantages qui justifieraient notre choix:

1. Un plus large champ d'action. Par conséquent, le lexicographe est libre de donner des analyses plus détaillées sur la question sans devoir se limiter à de simples traductions ou définitions comme le font la plupart des auteurs sur les proverbes. Il a ainsi la possibilité d'envisager l'aspect culturel du proverbe en même temps que les aspects sémantiques et formels.

2. Résoudre certains problèmes posés par le choix de l'ordre alphabétique. Par exemple, quel est le mot sous lequel il faut placer le proverbe, ou encore sur quel mot s'appuyer s'il faut placer le proverbe au début comme dans les dictionnaires spécialisés de proverbes. Réduire la présentation des données. Les proverbes sont groupés sous différents thèmes bien précis, ce qui a l'avantage d'en retracer plus facilement le sens contrairement au procédé qui consiste à les mettre sous un "motclé".

3. Enfin, se servir de la classification proposée par le dr Lisimba permettrait de restaurer la fonction du proverbe, c'est-à-dire savoir bien parler, et par là même, présenter le discours poétique de la littérature orale, discours souvent méconnu du public.

En conclusion, il nous semble que le but poursuivit dans la composition de dictionnaires en général est non seulement d'informer les usagers sur le langage de leur communauté, mais aussi de leur donner des armes pour en faire de bon citoyens, sachant s'exprimer et respectant les valeurs de la société. Bien plus qu'un ouvrage didactique, le dictionnaire, de part l'autorité qui lui est conférée, est un mode d'emploi pour l'intégration des individus dans une société. Dans les sociétés traditionnelles africaines, les proverbes étaient utilisés pour transmettre le savoir, la connaissance, la sagesse. Bien intégrer ces derniers dans l'usage du dictionnaire restituerait certaines valeurs morales cor- 
rompues depuis l'avènement de la modernisation, ce qui est un fléau parmi les jeunes de ces dernières décénies.

\section{Notes de références}

1. Parmis la variété de genres littéraires appartenant à la littérature orale, le proverbe est l'une des formes des plus pertinentes pour les temps modernes. Il est et demeure un puissant facteur de transmission de la culture, des valeurs sociales et morales, et des idées d'un peuple d'une géneration à une autre.

2. Horrifiée par la montée térrifiante de la violence, et cherchant un remède, je conclus que tout remède effectif devrait combiner diverses solutions. Le langage des proverbes m'apparaît alors comme l'un des instruments qui pourrait aider.

3. En Afrique du Sud, les peuples sont généralement pasteur ou fermier. La vache est source de richesse et est symbole de pouvoir et d'autorité. On retrouvera donc une forte concentration de proverbes l'ayant comme objet principal, tandis que dans les pays d'Afrique centrale comme le Gabon, elle serait quasi inexistante. L'autorité et le pouvoir seront symbolisés par les animaux de la forêt, tels que le lion ou le léopard.

4. Les proverbes sont cristallisés par les diverses expériences humaines. Parce qu'il n'existe pas d'unique solution aux problèmes des êtres humains, les proverbes n'offrent ni d'uniques, ni de stables solutions. (...) Ils présentent par contre des réponses et des conseils précis et prêts à être utilisés.

\section{Bibliographie}

\section{Dictionnaires}

Dubois, J. (Éd.). 1966. Dictionnaire du Français Contemporain. Paris: Larousse.

Maloux, M. 1980. Dictionnaire des Proverbes, Sentences et Maximes. Montréal: Larousse.

Rey, A (Éd.). 1988. Le Micro Robert de Poche: dictionnaire d'apprentissage de la langue française. Montréal: Icorobet.

\section{Autres lectures}

Akporobaro, F.P.O. et J.A. Emovon. 1994. Nigerian Proverbs: Meaning and Relevance Today. Lagos: Nigeria Magazine.

Dubois J. et C. Dubois. 1971. Introduction à la lexicographie: Le dictionnaire. Paris: Larousse.

Kuzwayo, E.K. 1998. African Wisdom. Cape Town: Kwela.

Lisimba, M. 1999. Kongo Proverbs and the Origins of Bantu Wisdom. Pésence Africaine.

Prinsloo, Anton F. 1997. Afrikaanse Spreekwoorde en Uitdrukkings. Pretoria: J.L.van Schaik.

Raponda-Walker, A. 1993. 3000 proverbes du Gabon. Paris: Saint-Paul.

Sabbagha, N.G. et M.S.B. Kritzinger. 1980. English Proverbs and Expressions with Afrikaans Equivalents. Pretoria: J.L. van Schaik. 